\title{
Shuffled-token Detection for Refining Pre-trained RoBERTa
}

\author{
Subhadarshi Panda \\ Graduate Center \\ CUNY \\ spanda@gc. cuny. edu
}

\author{
Jeewon Ha \\ New York University \\ jh6926enyu. edu
}

\author{
Benjamin Bloch \\ New York University \\ bb1976@nyu. edu
}

\begin{abstract}
State-of-the-art transformer models have achieved robust performance on a variety of NLP tasks. Many of these approaches have employed domain agnostic pre-training tasks to train models that yield highly generalized sentence representations that can be fine-tuned for specific downstream tasks. We propose refining a pre-trained NLP model using the objective of detecting shuffled tokens. We use a sequential approach by starting with the pre-trained RoBERTa model and training it using our approach. Applying random shuffling strategy on the word-level, we found that our approach enables the RoBERTa model achieve better performance on 4 out of 7 GLUE tasks. Our results indicate that learning to detect shuffled tokens is a promising approach to learn more coherent sentence representations. ${ }^{1}$
\end{abstract}

\section{Introduction}

The method of pre-training natural language models has been shown to greatly improve model performance on a wide range of NLP tasks (Peters et al., 2018; Radford et al., 2018; Howard and Ruder, 2018). State-of-the-art models that utilize transformers and deep bi-directional representations of text such as BERT, RoBERTa, and ALBERT (Devlin et al., 2019; Liu et al., 2019; Lan et al., 2020) have achieved superior results by pre-training on general, large corpora to learn rich representations from unlabeled data. Particularly helpful in low training data resource scenarios, unsupervised pretraining has become the first step for many language models to build powerful linguistic representations before fine tuning for downstream target tasks.

BERT style models use masked language modeling (MLM) and sometimes next sentence prediction, as pre-training tasks. While these tasks have

\footnotetext{
${ }^{1}$ The code is available at https://github.com/ subhadarship/learning-to-unjumble.
}

been shown to produce transferable sentence representations for many NLP tasks, using additional domain-agnostic pre-training tasks such as sentence shuffling may improve model performance. In a seminal cognitive psychology study it has been demonstrated that humans have a well trained ability to parse shuffled sentences (McCusker et al., 1981). Moreover, it has been shown that pre-trained models sometimes overlook word order while making predictions (Pham et al., 2020), and encouraging models to capture word order improves the classification performance. Shuffling as a pre-training task may therefore help expand transformer models to achieve even better performance on NLP tasks.

Drawing inspiration from recent work in reconstructing shuffled text (Lewis et al., 2020; Raffel et al., 2020), we propose that pre-training the RoBERTa model with a token modification discrimination head on randomly shuffled sentences provides constructive learning objective, which helps the model learn coherent representations and facilitate model recognition of the key pieces of a sentence and their association. To substantiate the argument, we design experiments to examine the model performance of RoBERTa with the proposed approach. The results demonstrate that pre-training the model with shuffled sentences enhances the scores of a majority of GLUE tasks.

\section{Related Work}

Shuffling sentences and words has often been used as a downstream task to evaluate model performance. One relevant example is the work by Sakaguchi et al. (2017) to develop a semi-character RNN model that surpasses previous spell-check methodologies on the Cmadbrigde Uinervtisy effect, where humans can easily reconstruct the shuffled token. Yang and Gao (2019) explored the performance of BERT on a shuffled sentence downstream task and highlighted some induced bias in the model that is the cause of incorrect predictions 


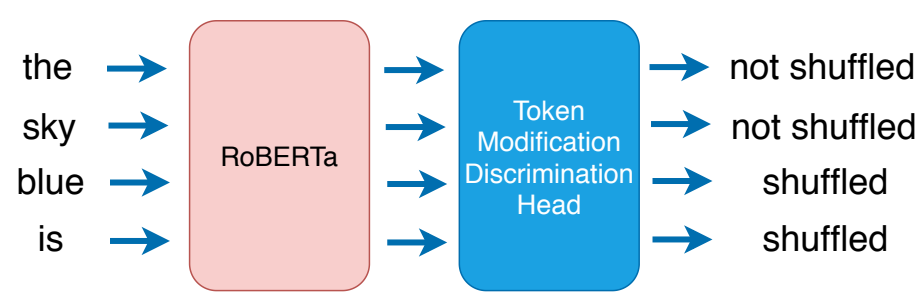

Figure 1: Illustration of our model for detecting shuffled tokens. The original sentence is "the sky is blue".

for noisy inputs. While the authors propose removing the induced bias from the representations to improve results, they do not consider the possibility of pre-training the model with shuffled sentences.

The use of un-ordered or noisy data in model training itself has proven effective. A number of studies have focused on using shuffled input to create useful sentence representation vectors for language models. Kiros et al. (2015) developed the skip-thoughts method to accomplish the task of reconstructing sentence order from a shuffled input. The authors used an encoder-decoder RNN model at the sentence level that allows a sentence to predict the adjacent sentences. Logeswaran et al. (2016) explored how sentence ordering tasks can help models learn text coherence. Using an RNN based approach, they train models to identify the correct ordering of sentences and show that models learn both document structure and useful sentence representations during this task. Jernite et al. (2017) employed discourse based learning objectives to help models understand discourse coherence. Specifically, given some sentences, they ask the model to predict if the sentences are in order, or if one sentence comes next to a set of sentences, or to predict the conjunction that joins the sentences. They showed that using these objectives to train models achieves significant reduction in computational training costs and is also effective when using unlabeled data.

There are a number of papers that focus on wordlevel shuffling, as opposed to sentence-level shuffling. Hill et al. (2016) developed the Sequential Denoising Autoencoder (SDAE) method, where a sentence is corrupted using a noise function determined by free parameters. After a certain percentage of words have been corrupted, an LSTM encoder-decoder model is tasked with predicting the original sentence from the corrupted version. The authors demonstrate training with noisy inputs allowed SDAE to significantly outperform regular SAE models, which did not introduce word-level- noise factors.

One closely related paper in the field of computer vision leverages the use of shuffled input in model training. Noroozi and Favaro (2016) employ a CNN model that is trained to solve jigsaw puzzles to determine correct spatial representation. Their results show that using shuffled input helps models learn that images are made up of different parts, and their relationship to the whole.

Finally, a variety of studies demonstrate that further pre-training performed after the general purpose BERT pre-training leads to better model results instead of simply fine-tuning downstream. Domain specific pre-training, such as BioBERT (Lee et al., 2019), story ending prediction by TransBERT (Li et al., 2019), and video caption classification by videoBERT (Sun et al., 2019) are all examples where expanding the pre-training tasks for BERT has achieved enhancement in model performance. TransBERT in particular demonstrates that further pre-training using targeted supervised tasks achieves better results than relying only on the unsupervised pre-training in BERT.

\section{Methodology}

Consider a sequence of tokens $\boldsymbol{x}$. We first obtain $\boldsymbol{x}^{\text {shuffled }}$ from $\boldsymbol{x}$ by shuffling a set of tokens of $\boldsymbol{x}$. Given $\boldsymbol{x}^{\text {shuffled }}$, we detect if tokens are shuffled or not by using a token modification discrimination head on top of the RoBERTa base model. Our choice of the discriminative head is motivated by the recent success of ELECTRA (Clark et al., 2019).

\subsection{Creating Shuffled Tokens for Training}

We permute text sequences at the word level based on a probability $p$. We consider shuffling on a word level rather than a sub-word level. One straightforward approach to achieve is to create the shuffled tokens from a sequence and then use RobertaTokenizer to tokenize the shuffled sequence. However, this approach is problematic since the number of sub-words after tokenization 


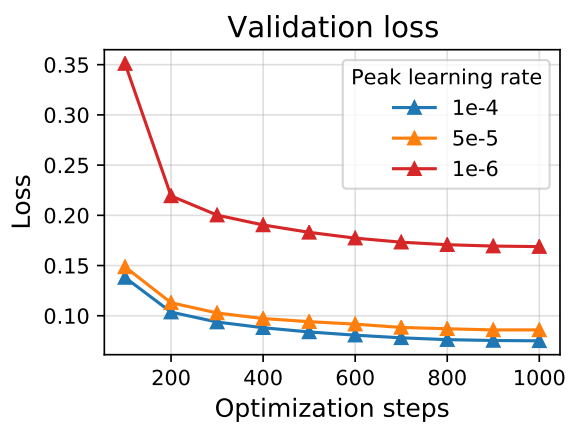

Figure 2: Validation loss as training progresses.

may differ between the original and the shuffled sentence. In order to ensure that the sub-words belonging to a word stay intact and are not shuffled away, we create a mapping, which maps each subword to the corresponding word. Then, we tokenize the original sequence and shuffle the tokens based on the mapping so that all the sub-words belonging to a word occur together. Further, we define the target tensor which has binary labels for each token that specifies whether the token was shuffled or not.

\subsection{Shuffling Strategy}

We randomly permute the words in a sequence based on a probability $p$ for our experiments highlighted in Section 4. Note that fraction $\geq p$ of the input tokens would be shuffled since one or more input tokens (sub-words) belong to a single word.

\subsection{RoBERTa Model with Token Modification Discrimination Head}

Figure 1 shows an overview of our complete model. We use the RoBERTa model to map a sequence of input tokens $\boldsymbol{x}^{\text {shuffled }}=\left[x_{1}, \ldots, x_{n}\right]$ into a sequence of contexualized vectors $h(\boldsymbol{x})=$ $\left[h_{1}, \ldots, h_{n}\right]$. We add a token modification discriminator head to classify each hidden representation $h_{i}$ to 0 (if the token at $i$-th place is not shuffled) or 1 (if the token at the $i$-th place is shuffled). Specifically, the head contains two linear layers with parameters $\left\{W_{A}\right\}$ and $\left\{W_{B}\right\}$. First, for every hidden vector $h_{i}$, we compute $h_{i}^{\prime}=\operatorname{GELU}\left(W_{A}^{T} h_{i}\right)$ where the GELU activation function (Hendrycks and Gimpel, 2016) is used. Then, we compute the output of the model $D\left(\boldsymbol{x}^{\text {shuffled }}, i\right)=\sigma\left(W_{B}^{T} h_{i}^{\prime}\right)$. During training, we minimize the sum of the binary cross entropy loss for every token.

$$
\begin{gathered}
\mathcal{L}(\boldsymbol{x}, \theta)=\mathbb{E}\left(\sum_{i=1}^{n}-\mathbb{1}\left(x_{i}^{\text {shuffled }}=x_{i}\right) \log D\left(\boldsymbol{x}^{\text {shuffled }}, i\right)\right. \\
\left.-\mathbb{1}\left(x_{i}^{\text {shuffled }} \neq x_{i}\right) \log \left(1-D\left(\boldsymbol{x}^{\text {shuffled }}, i\right)\right)\right)
\end{gathered}
$$

\section{Experiments}

\subsection{Baseline}

As our baseline approach, we trained the RoBERTa base model with the token modification discrimination head for detecting masked tokens instead of detecting shuffled tokens. The baseline training was done for the same number of optimization steps as the proposed approach for a fair comparison.

\subsection{Dataset for Shuffled-Token Detection}

We extracted $133 \mathrm{~K}$ articles from Wikidump. ${ }^{2}$ We used each paragraph in the extracted text as a data sample for our model. We filtered out samples that were either spaces-only or had more than 512 tokens after tokenizing with the pretrained RobertaTokenizer of the roberta-base model. We finally randomly split the samples into $1.3 \mathrm{M}$ for training and $14 \mathrm{~K}$ for validation.

Dataset for masked token detection We used the same Wikidump dataset for the baseline approach as well, where we continue training pretrained RoBERTa on the objective of detecting masked tokens.

\subsection{Implementation}

We built our model using HuggingFace transformers (Wolf et al., 2020). All experiments have been performed using the RoBERTa base model with the token modification discrimination head described in Section 3.3.

The hyperparameters used in our experiments follow the hyperparameters of the RoBERTa base model except for the warmup steps, batch size, peak learning rate, and the maximum training steps. For our experiments, we use 100 linear warmup steps followed by linear decay of the learning rate outlined in Figure 3.

To find the optimal peak learning rate and the maximum steps, we performed a hyperparameter search over the learning rates $\{1 \mathrm{e}-4,5 e-5,1 e-6\}$

\footnotetext{
${ }^{2}$ Timestamp May 9th, 2020. We used the scripts from https://github.com/ NVIDIA/DeepLearningExamples/tree/ master/PyTorch/LanguageModeling/BERT\# getting-the-data to extract the data.
} 


\begin{tabular}{|c|c|c|c|c|c|c|c|}
\hline Task $\rightarrow$ & CoLA & SST-2 & MRPC & STS-B & QNLI & RTE & WNLI \\
\hline Metric $\rightarrow$ & Matthew's corr. & Accuracy & F1 score & Spearman corr & Accuracy & Accuracy & Accuracy \\
\hline Plain pre-trained RoBERTa & 0.557 & 0.946 & 0.901 & 0.896 & 0.928 & 0.661 & 0.423 \\
\hline Masked-token detection (Baseline) & 0.508 & 0.950 & 0.869 & 0.888 & 0.924 & 0.631 & 0.563 \\
\hline Shuffled-token detection & 0.621 & 0.92 & 0.905 & 0.886 & 0.928 & 0.704 & 0.437 \\
\hline
\end{tabular}

Table 1: Results on GLUE tasks.

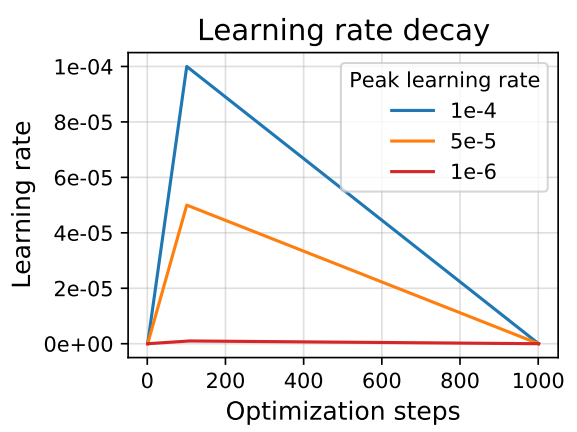

Figure 3: Learning rate as training progresses.

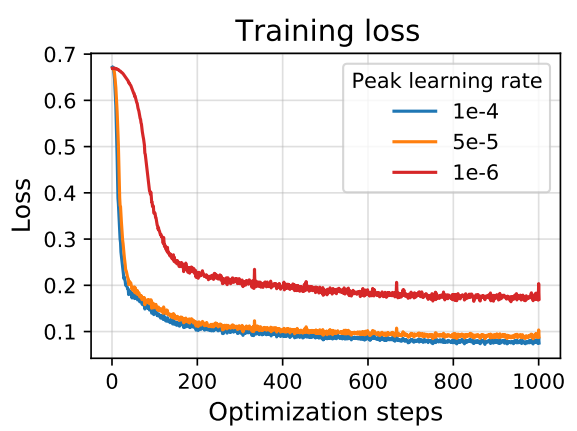

Figure 4: Training loss logged after every training step.

and over the maximum steps from $[100,1000]$ with a step size of 100 . Changes in learning rate with the increase in optimization steps for different peak learning rates are shown in Figure 3. The results for the validation loss with an increasing number of optimization steps for the different learning rates is illustrated in Figure 2. The training loss is outlined in Figure 4. We observe that the minimum training loss, as well as validation loss, are achieved with the peak learning rate of 1e-4. Moreover, the training loss and the validation loss keep on decreasing with the number of optimization steps continuously till 1000 steps which shows that training the model for more number of steps could be beneficial. The optimal maximum steps as shown in Figure 4 and 2 is $1000 .^{3}$ For training our baseline approach of detecting mask tokens, we set the learning rate to $1 \mathrm{e}-4$.

\footnotetext{
${ }^{3}$ An actual optimum number of steps could be more than 1000 and training further would give us the best value for the maximum steps.
}

The probability of masking tokens (sub-words) in the baseline approach was fixed to 0.15 as done in previous work (Devlin et al., 2019; Liu et al., 2019). For the proposed approach, we also set the probability $p$ of shuffling tokens (words) to 0.15 .

On using large batch sizes Pre-training procedures have been shown to be effective when using large batch sizes (Liu et al., 2019). Training our model directly on a very large batch size required computation power beyond what was available. To alleviate this problem, we used gradient accumulation for 64 steps with a per GPU batch size of 16. We used distributed training on 4 Nvidia K80 GPUs to train our models. The effective batch size during training was 4096.

\subsection{Downstream Evaluation}

We evaluate our approach on 7 GLUE tasks using the metrics outlined in Table 1 . We use the same set of hyperparameters for fine-tuning for downstream tasks for each approach for a fair comparison. Methods for comparison to our approach include (a) the baseline approach where the training objective is detecting masked tokens, and (b) the plain pre-trained RoBERTa base model. The values of hyperparameters used for GLUE fine-tuning are outlined in Table 2. The rest of the hyperparameters are set to default values. ${ }^{4}$

\begin{tabular}{c|c} 
Hyperparameter & Value \\
\hline Maximum Sequence Length & 128 \\
Batch Size & 64 \\
Learning Rate & $2 \mathrm{e}-5$ \\
Number of epochs & 3
\end{tabular}

Table 2: Hyperparameters for fine-tuning RoBERTa model.

\subsection{Results and Analysis}

Table 1 presents the results for the 7 GLUE tasks. Our model trained to detect randomly shuffled to-

\footnotetext{
${ }^{4}$ The default hyperparameters are as in https: //github.com/huggingface/transformers/ blob/v2.8.0/examples/run_glue.py.
} 
kens performs the best in 4 of the 7 downstream tasks, namely CoLA, MRPC, QNLI and RTE. The scores for the baseline, where the objective is to detect masked tokens, are interestingly sometimes worse than the plain pre-trained RoBERTa's scores. For example, the CoLA score using plain pre-trained RoBERTa is 0.557 whereas the score obtained by the baseline is 0.508 .

The model performance based on the proposed approach on individual tasks gives us insights about what aspects of natural language our model improved in learning. Our model's performance on CoLA, which predicts grammatical correctness of a sentence, is better, indicating that the pre-training task may have enhanced the model's ability to learn grammatical information. Moreover, better performance on RTE, MRPC and QNLI shows that with the proposed approach, the model better understands the semantic relationships such as similarity and entailment.

However, random shuffling hurts the performance of the model on WNLI significantly in comparison to the baseline. This may be due to the fact that WNLI forms a pair of sentences by replacing the ambiguous pronouns with their referents. Since we are shuffling the words, it is likely that the nouns will be shuffled, resulting in misleading replacement of the ambiguous pronoun.

Our baseline model outperforms the shuffledtoken detection approach on SST-2 task which predicts the sentiment polarity of the movie reviews. One possible explanation is that shuffling negations in presence of contrasting conjunctions can significantly change the sentiment associated with the sentence. $^{5}$

\section{Conclusion and Future Work}

In this paper, we examine the performance of RoBERTa model with token modification discrimination head on detecting randomly shuffled tokens. We have demonstrated that detecting shuffled tokens is indeed a challenging yet advantageous task, which allows the model to learn coherent representations of the sentences. In this work, we start with pre-trained RoBERTa base model and train it further on the shuffled token detection task.

For future work, the model can be further explored by expanding the shuffling strategy. One possible strategy is part of speech (POS) shuffling,

\footnotetext{
${ }^{5}$ For instance, consider the sentence "That movie was good but I did not watch it." A random shuffled sentence can be "The movie was not good but I did watch it."
}

which randomly permutes specific POS tokens such as nouns or verbs. Instead of detecting shuffled tokens, another objective would be to predict the original positions of the shuffled tokens. Yet another objective that can be explored is combining our proposed loss with the masked language modeling loss. We would also like to study our approach when applied to other pre-trained models such as ALBERT and ELECTRA.

\section{Acknowledgments}

We thank Sam Bowman for friendly discussions. We also thank the anonymous reviewers for the useful comments and suggestions.

\section{References}

Kevin Clark, Minh-Thang Luong, Quoc V Le, and Christopher D Manning. 2019. Electra: Pre-training text encoders as discriminators rather than generators. In International Conference on Learning Representations.

Jacob Devlin, Ming-Wei Chang, Kenton Lee, and Kristina Toutanova. 2019. BERT: Pre-training of deep bidirectional transformers for language understanding. In Proceedings of the 2019 Conference of the North American Chapter of the Association for Computational Linguistics: Human Language Technologies, Volume 1 (Long and Short Papers), pages 4171-4186, Minneapolis, Minnesota. Association for Computational Linguistics.

Dan Hendrycks and Kevin Gimpel. 2016. Gaussian error linear units (gelus). arXiv preprint arXiv:1606.08415.

Felix Hill, Kyunghyun Cho, and Anna Korhonen. 2016. Learning distributed representations of sentences from unlabelled data. In Proceedings of the 2016 Conference of the North American Chapter of the Association for Computational Linguistics: $\mathrm{Hu}$ man Language Technologies, pages 1367-1377, San Diego, California. Association for Computational Linguistics.

Jeremy Howard and Sebastian Ruder. 2018. Universal language model fine-tuning for text classification. In Proceedings of the 56th Annual Meeting of the Association for Computational Linguistics (Volume 1: Long Papers), pages 328-339, Melbourne, Australia. Association for Computational Linguistics.

Yacine Jernite, Samuel R. Bowman, and David A. Sontag. 2017. Discourse-based objectives for fast unsupervised sentence representation learning. CoRR, abs/1705.00557.

Ryan Kiros, Yukun Zhu, Russ R Salakhutdinov, Richard Zemel, Raquel Urtasun, Antonio Torralba, and Sanja Fidler. 2015. Skip-thought vectors. In C. Cortes, N. D. Lawrence, D. D. Lee, M. Sugiyama, 
and R. Garnett, editors, Advances in Neural Information Processing Systems 28, pages 3294-3302. Curran Associates, Inc.

Zhenzhong Lan, Mingda Chen, Sebastian Goodman, Kevin Gimpel, Piyush Sharma, and Radu Soricut 2020. Albert: A lite bert for self-supervised learning of language representations. In International Conference on Learning Representations.

Jinhyuk Lee, Wonjin Yoon, Sungdong Kim, Donghyeon Kim, Sunkyu Kim, Chan Ho So, and Jaewoo Kang. 2019. BioBERT: a pretrained biomedical language representation model for biomedical text mining. Bioinformatics, 36(4):1234-1240.

Mike Lewis, Yinhan Liu, Naman Goyal, Marjan Ghazvininejad, Abdelrahman Mohamed, Omer Levy, Veselin Stoyanov, and Luke Zettlemoyer. 2020. BART: Denoising sequence-to-sequence pretraining for natural language generation, translation, and comprehension. In Proceedings of the 58th Annual Meeting of the Association for Computational Linguistics, pages 7871-7880, Online. Association for Computational Linguistics.

Zhongyang Li, Xiao Ding, and Ting Liu. 2019. Story ending prediction by transferable bert. In Proceedings of the Twenty-Eighth International Joint Conference on Artificial Intelligence, IJCAI-19, pages 1800-1806. International Joint Conferences on Artificial Intelligence Organization.

Yinhan Liu, Myle Ott, Naman Goyal, Jingfei Du, Mandar Joshi, Danqi Chen, Omer Levy, Mike Lewis, Luke Zettlemoyer, and Veselin Stoyanov. 2019. Roberta: A robustly optimized BERT pretraining approach. CoRR, abs/1907.11692.

Lajanugen Logeswaran, Honglak Lee, and Dragomir R. Radev. 2016. Sentence ordering using recurrent neural networks. CoRR, abs/1611.02654.

Leo McCusker, Philip Gough, and Randolph Bias. 1981. Word recognition inside out and outside in. journal of experimental psychology: Human perception and performance. 7(3):538-551.

Mehdi Noroozi and Paolo Favaro. 2016. Unsupervised learning of visual representations by solving jigsaw puzzles. In Computer Vision - ECCV 2016, pages 69-84, Cham. Springer International Publishing.

Matthew Peters, Mark Neumann, Mohit Iyyer, Matt Gardner, Christopher Clark, Kenton Lee, and Luke Zettlemoyer. 2018. Deep contextualized word representations. In Proceedings of the 2018 Conference of the North American Chapter of the Association for Computational Linguistics: Human Language Technologies, Volume 1 (Long Papers), pages 2227-2237, New Orleans, Louisiana. Association for Computational Linguistics.
Thang M. Pham, Trung Bui, Long Mai, and Anh Nguyen. 2020. Out of order: How important is the sequential order of words in a sentence in natural language understanding tasks?

Alec Radford, Karthik Narasimhan, Tim Salimans, and Ilya Sutskever. 2018. Improving language understanding by generative pre-training.

Colin Raffel, Noam Shazeer, Adam Roberts, Katherine Lee, Sharan Narang, Michael Matena, Yanqi Zhou, Wei Li, and Peter J. Liu. 2020. Exploring the limits of transfer learning with a unified text-totext transformer. Journal of Machine Learning Research, 21(140):1-67.

Keisuke Sakaguchi, Kevin Duh, Matt Post, and Benjamin Van Durme. 2017. Robsut wrod reocginiton via semi-character recurrent neural network. In Proceedings of the Thirty-First AAAI Conference on Artificial Intelligence, AAAI'17, page 3281-3287. AAAI Press.

Chen Sun, Austin Myers, Carl Vondrick, Kevin Murphy, and Cordelia Schmid. 2019. Videobert: A joint model for video and language representation learning. In Proceedings of the IEEE/CVF International Conference on Computer Vision (ICCV).

Thomas Wolf, Lysandre Debut, Victor Sanh, Julien Chaumond, Clement Delangue, Anthony Moi, Pierric Cistac, Tim Rault, Rémi Louf, Morgan Funtowicz, Joe Davison, Sam Shleifer, Patrick von Platen, Clara Ma, Yacine Jernite, Julien Plu, Canwen Xu, Teven Le Scao, Sylvain Gugger, Mariama Drame, Quentin Lhoest, and Alexander M. Rush. 2020. Transformers: State-of-the-art natural language processing. In Proceedings of the 2020 Conference on Empirical Methods in Natural Language Processing: System Demonstrations, pages 38-45, Online. Association for Computational Linguistics.

Runzhe Yang and Zhongqiao Gao. 2019. Can machines read jmulbed senetcnes? 